\title{
Study on the Low Energy Consumption Method for Light-Wight Devices in IoT Service Environment
}

\author{
Young-Hyun Kim ${ }^{1}$, Myong-Soo Kim ${ }^{2}$, Myung-Hye Park ${ }^{3}$, Soo-Kyung Kang ${ }^{4}$ and \\ Chang-Soo Eun ${ }^{5 *}$ \\ ${ }^{1,2,3,4}$ Korea Electric Power Corporation \\ ${ }^{5}$ Chungnam National University \\ 1younghyun.kim@kepco.co.kr, ${ }^{2}$ myongsoo.kim@kepco.co.kr, \\ ${ }^{3}$ myunghye.park@kepco.co.kr, ${ }^{4}$ sk.kang@kepco.co.kr, ${ }^{5}$ eun@cnu.ac.kr
}

\begin{abstract}
As the interest in the 4th industrial revolution continues, there is also the growing interest in infrastructure related technology. One of them is the need for Internet of Things (IoT) that can exchange information between various terminals. IoT technology is evolving the existing M2M system to provide low energy consumption and light weight by providing a wider coverage. Also interoperability between manufacturers should be ensured through a standard acceptance. The proponents of this paper propose a low energy consumption method based on LwM2M standard which is the standard technology representative of IoT. The designed system proved that it can save energy compared to the existing IoT device through the operation algorithm such as optimized event-driven method and parallel processing method.
\end{abstract}

Keywords: IoT, Lightweight Devices, LwM2M, Energy Consumption

\section{Introduction}

Internet of Things(IoT) refers to an infrastructure in which intelligent objects are networked to exchange mutual information between people and objects, objects and objects, and intelligent services combined with knowledge based on context awareness. [1]. Various technologies have been developed, proved and commercialized in order to build a stable infrastructure and provide services. As the ecosystem in IoT is formed, interoperability between products will also become an important issue.

As devices in IoT are being operated in the outdoor field, it is highly required for lightweight devices powered by small battery and resource-constrained HW to design and implement a protocol in an energy efficient manner. Accordingly, the solution for the above problem in the field is being discussed as the key to success of the IoT market. In this paper, a protocol operation and operation optimization method was proposed based on the LwM2M international standard which is most popular in IoT.

The remainder of this paper is organized as follows: Section 2 describes the related technologies needed for the analysis of this paper. Section 3 presents a method for satisfying the requirements of low energy consumption under lightweight devices, which are real world requirements. In Section 4, the performance of the proposed algorithm is verified. Finally, Section 5 concludes the paper.

Received (May 18, 2018), Review Result (July 28, 2018), Accepted (August 8, 2018)

* Corresponding Author 


\section{Related Work}

\subsection{IoT System}

The necessary technology for building the IoT can be divided into the technical fields for providing the technology and services required for infrastructure construction and operation. The construction and operation technology includes communication technology, information interlocking technology, and management technology that can gather information to the upper server level centering on the terminal where the information is generated. This includes devices on the site, a gateway for connecting them to the platform, and the platform to provide services. [2] [5]. This is shown in Figure 1.

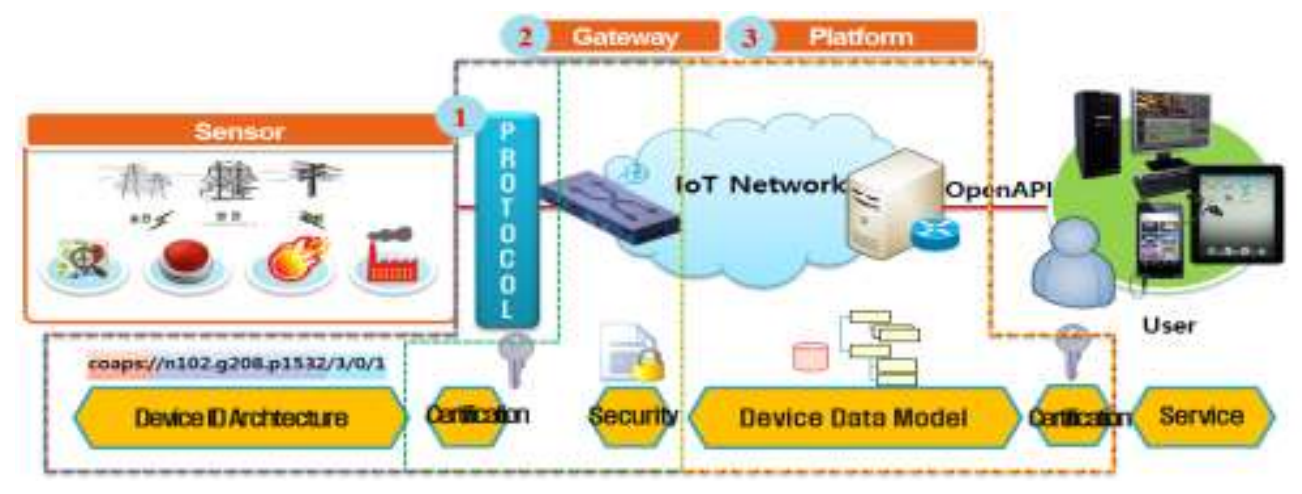

Figure 1. IoT System Architecture

-Device: Protocol interface design that accommodates various sensors and terminals

Gateway: Data collection, real-time processing, and mutual link utilization

·Platform: Open API design, object data analysis, security and visualization

\subsection{LwM2M}

In order to send and receive information between IoT devices, information modeling at the upper layer and standardization are required in order to ensure interoperability between various manufacturers. In the IoT field, LwM2M technology exists as a device management standard protocol designed for sensor networks. It was developed to demand in the market for a common standard for managing lightweight and low power devices on a variety of networks necessary to realize the potential of IoT. The LwM2M protocol, designed for remote management of M2M devices and related service enablement, features a modern architectural design based on REST, defines an extensible resource and data model, and builds on an efficient secure data transfer standard called the Constrained Application Protocol (CoAP). This technology has been adopted as a technology capable of efficiently managing multiple devices in the OneM2M standard organization and has been utilized in various service sites [3] [4].

The LWM2M protocol registers the device with the gateway using the CoAP Resource Directory. After registration, the device information will be maintained through periodic update.

The gateway that controls device information is registered with the platform. The standard is available with oneM2M or LWM2M. After registration, the gateway performs periodic update to maintain the gateway and the device information. In order to periodically report the information, the gateway subscribes to the device information and periodically reports a notify message to the IoT gateway based on 
the registered subscribe information. The gateways report information received from the devices to the platform periodically, and can also request information directly in addition to reporting periodic information. The gateway receiving the information inquiry request from the platform can request the corresponding device again to acquire the information, and if it cannot directly request the device, it responds with the cached information. A control operation was also included that can request a specific operation or modify the information similarly to the information query.

Table 1 shows the standard that reflects the protocol definition between the devices- gateway and the gateway-IoT platform.

Table 1. Related Standards in IoT

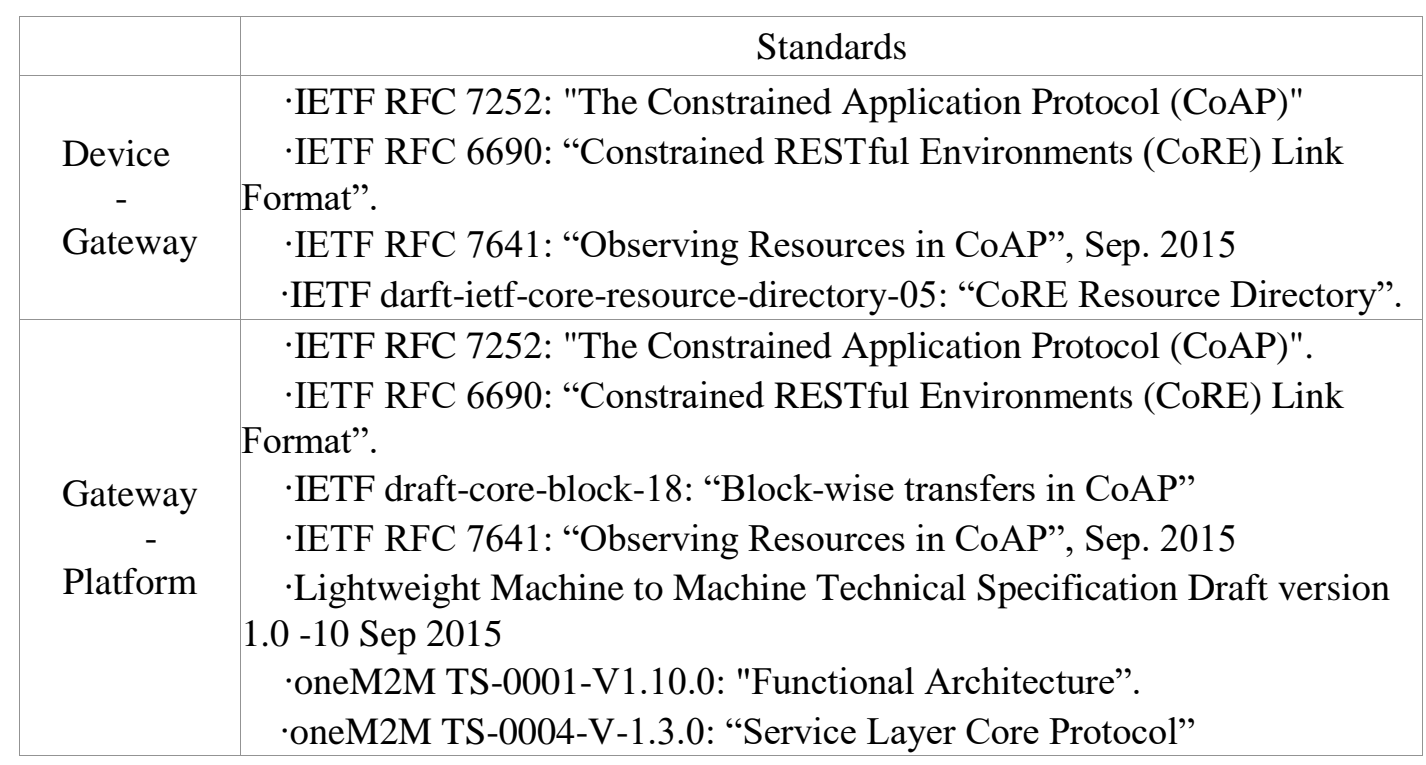

The LwM2M standard defines OID-based identifiers for the purpose of identifying the gateway and the device and the resource profile representing the meaning of the resource in the IoT device. The device identifier consists of Device Indication ID, Service ID, Standard ID, Manufacturer ID, Model ID, and Serial ID. In the resource profile identifier, an ID that can describe the characteristics of the resource is described in the OID system and included in oneM2M-based resource model. The structure of Resource Profile ID consists of Resource Profile Indication ID, Object ID, and Resource ID. For example, ontologyRef attribute was used to indicate the meaning of the actual data in the <container> resource, and the resource profile ID will be displayed in this information.

\section{System Design}

In this section, an energy efficient system that can guarantee interoperability of IoT devices and can be used in the field through low energy consumption operation was proposed. In order to ensure interoperability between devices, it is necessary to adopt the most recognizable and widely used standard technology hence, the LwM2M standard was adopted. Based on this, technical proposals for improvement measures were explained by simple registration/simple reporting and optimization of operation method.

\subsection{Operation Method based on LwM2M}

In order to meet the low-speed, low-power and low-cost requirements defined above, it is necessary to minimize the amount of information to be transmitted and the number of 
communications. In contrast, the established standards define a complex registration process to accommodate a variety of manufacturers and devices. One of the examples is that the device for registration must know the gateway IP address, port number, and path information for the resource information. When the device accesses the gateway for registration, a Resource Directory (RD) search is performed, and the resource information of the device is registered in the gateway using the obtained information. [7]. It needs to communicate between them 6 times. To simplify the operation of the device, a simple registration and a simple reporting scheme were proposed. This technique is that the platform stores previously determined information such as terminal name, memory size, manufacturer, sensor type, etc. The device transmits only information that can be changed when it is installed and registered in the actual field, thereby minimizing the transmission / reception information transmission amount. This is explained as follows.
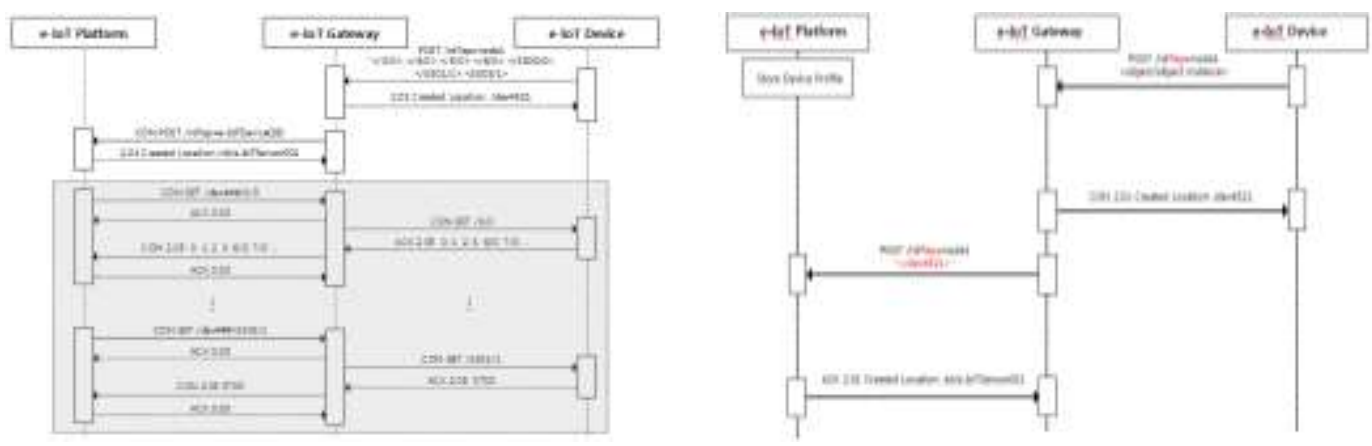

\section{Figure 2. Registration Procedure: (Left) General Approach, (Right) Simple Process}

3.1.1. Simple Registration: Figure 2 shows the simple registration of a device with the name node1 to the gateway and the platform. Prior to performing the simple registration process, the device's predefined information is stored on the platform. Then, the device requests the POST message to the gateway by including the <object / object instance> list information in link-format in the registration request message and setting the end point name as the lep option. The gateway receiving the registration post message determines from the lep option if it is a simple registration process and generates the registered location information. As a result of searching based on the lep information (device OID), if there is no stored information, the gateway requests the device's predefined information to the platform by further including a GET option in the process of performing a simple registration process with the platform. In this case, the simple registration message that is requested to the platform includes the location information generated by the gateway in the payload and delivers it to the platform. As a result, the gateway obtains the device's advance information and performs simple registration to the previously created location ("/ dev / 4521"). In this case, '4521' is a random number generated by the gateway RD. If the registration process has been performed through a plurality of connection settings, the operation will be improved by completing the registration with one packet in the case of this method.

3.1.2. Simple Reporting: Sensor-based devices that were used in the field have been developed and used as multi-function devices by incorporating various sensors such as temperature, humidity, and acceleration in one device. In this case, the periodic report collects the sensor information individually through the COAP Observer function for each sensor. A simple reporting method was proposed to transmit the measured values of various sensors installed in the device to the upper layer at a time. The simple report defines a virtual composite sensor that can represent measurement data of various physically existing 
sensors, and transmits data through it. Also, it defines the measurement value, unit and sensor quantity of the sensors constituting the composite sensor per resource, defines the ID to distinguish the sensors, and uses ';' as the delimiter.

Figure 3 shows an example of periodically reporting sensor values as composite data through the proposed method. Assuming that the complex sensor will be defined as '30000', the complex data structure will be operated in the following form through the simple registration form for ' $30000 / 0 / 1$ '. The device transmits the information in the following format, and transmits the sample information of the temperature sensor at 3303, the voltage sensor at 3316, and the current sensor at 3317 :

$$
\text { “/3303/0/5700;/dev/e1234/3316/0/5700;/dev/e1235/3317/0/5700” }
$$

Furthermore, when periodic reporting of setting CoAP Observe to "30000/0/0" will be performed, data will be transmitted at "37; 220;60". As a result of simple reporting, several packets were integrated into one and the RF transmission energy can be greatly reduced.

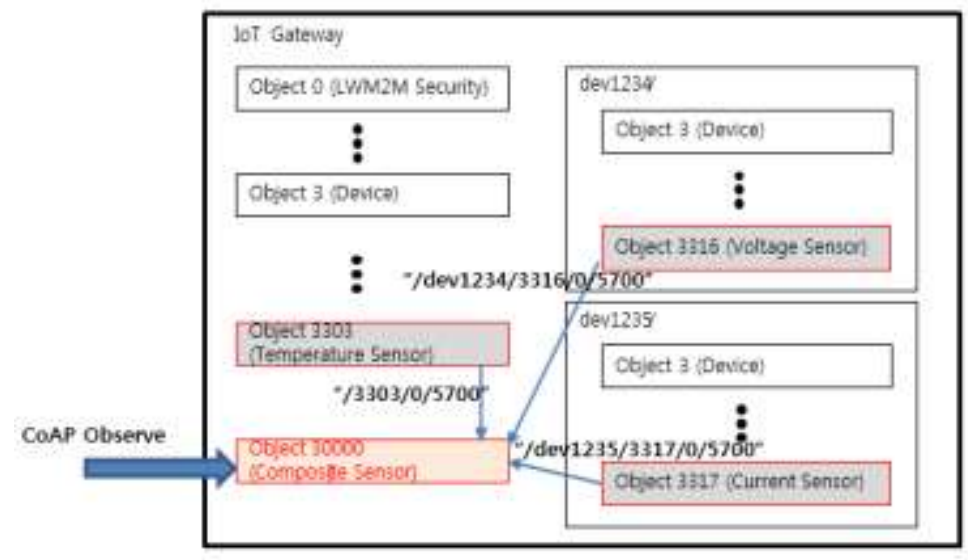

Figure 3. Information Modeling for Composite Sensor

\subsection{Sensor Operation}

Even when the number of information to be transmitted in the sensor is minimized, the device operates in a normal operation mode and consumes unnecessary power. IoT devices does not lots of communication such as 1 time / 1 hour, 1 time / 1 day. Therefore, there were many limitations in controlling the power of devices operating with battery even if the proposed method was used [8]. To solve this problem, an event-driven operating structure considering characteristics of IoT devices was proposed.

The IoT device has a characteristic of performing work according to periodic sensor signal processing. Accordingly, this proposed method of event-driven structure will generate a new task through HW Interrupt (Sleep, Wakeup), and the created Task will be stored in the FiFo Task Queue. The scheduler will execute the tasks stored in the FiFo Task Queue in order, and the executed Task can create a new Task, and a function to prevent duplicate storage of the created Task can be added. All tasks of the FiFo Task Queue will be executed and will enter HW Sleep if the state is empty. HW Sleep can minimize energy consumption by putting all hardware functions to sleep except RTC.

The operating method is divided into RUN (no sleep) and Sleep according to the provided service, and Sleep can be subdivided into Sleep, LP-Sleep, and Stop modes according to control items. The features of each mode are as follows. 


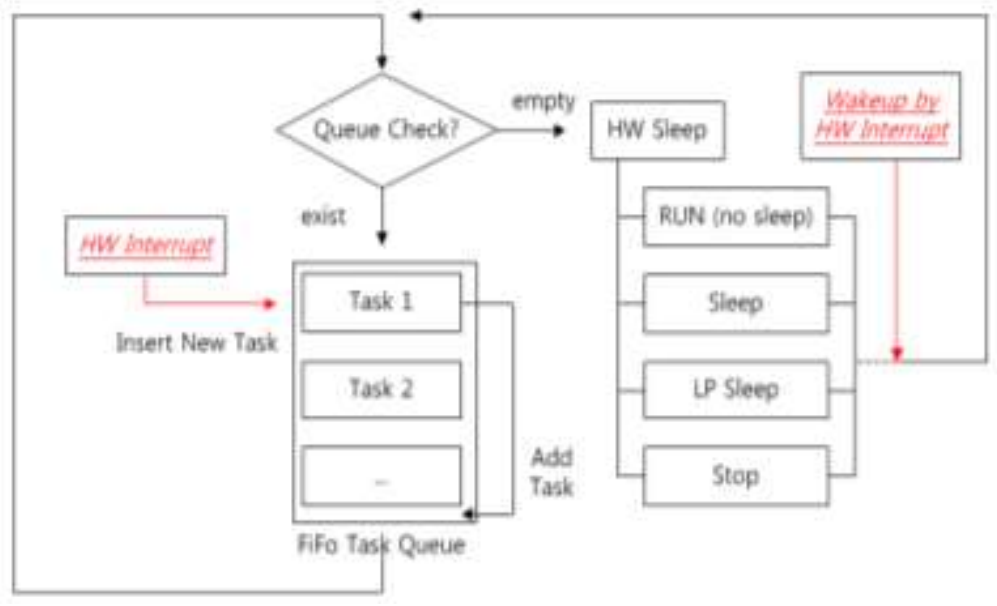

Figure 4. Event Driven Operation Method

The RUN mode is the stage where all functions can be utilized through continuous operation, and the power consumption is the largest at this time. In sleep mode, only the CPU sleeps and all clocks are turned on to reduce the power consumption compared with the run mode, and provide a step that can run quickly. When the device is divided into MCU, communication part and sensor part, (1) MCU generates signal processing and packet, (2) communication part uses data, and then it defines operation, (1)if it is in RUN mode, (2)or if it is in sleep Mode.

In the sleep mode defined above, a large number of peripherals can be operated, resulting in a large current consumption. In order to prevent this, stop mode was defined, but in the case of stop, there is a delay in service provision due to the waiting time when the operation is switched to the stop after the stop. LP-Sleep is a mode that operates at the lowest possible power level and at the same time, it can operate wakeup first. LP-Sleep task was divided into general task and Wakeup Task. In LP-Sleep operation mode, Wakeup Task is the first to be processed, and then the scheduler can set and operate a high priority level.

When the task creation, the task processing, and the sleep process defined above were performed, the processing of various sensors mounted on the device cannot be processed sequentially. Since each sensor has some hardware operation time for sensing, it takes a lot of time and power to process it sequentially. However, in this configuration where eventdriven structure was applied, the sensor processing task was executed sequentially, and then the CPU immediately sleeps, and the result processed by the sensor can be processed in parallel by generating and processing a task through an interrupt. That is, through the Run mode defined above, it is possible to simultaneously execute several sensors regardless of the hardware operation time of each sensor, and to shorten the sensor processing time and power consumption by putting the CPU to sleep when the defined task was completed. The processed information will be transmitted through the RF communication module. In the conventional operation method, as shown in Fig. 8, the status will be checked by sending a consecutive completion confirmation request to confirm completion of transmission / reception of RF. After confirming completion of RF operation through this polling method, the CPU could be made to sleep. However, in the event-driven structure, after executing a task to set up RF sending / receiving operation.

\section{Performance Evaluation}

In this section, the performance about the proposed algorithm was evaluated. M0 - based CPU was used and LWM2M was implemented based on the proposed OS. The sensor was equipped with 5 kinds of sensors, processing the sensing information, and transmitting it through the LoRa communication method. To compare the energy usage, the proposed device was measured against the general IoT devices. 
Table 2. Comparison of CPU Specification on the Devices

\begin{tabular}{|c|c|c|}
\hline & Generic IoT & Proposed IoT \\
\hline MCU & TI, MSP430F5419A & STM, STM32L072 \\
\hline Core & RISC 16bit $(1996$ release $)$ & ARM 32bit $(2009$ release $)$ \\
\hline Clock & $32.768 \mathrm{KHz}->1 \mathrm{M} / 2 \mathrm{MHz}$ & $16 \mathrm{M} / 32 \mathrm{MHz}$ \\
\hline Flash & $128 \mathrm{Kbytes}$ & $128 \mathrm{Kbytes}$ \\
\hline RAM & 16 Kbytes & $20 \mathrm{Kbytes}$ \\
\hline Price & $2.75 \$-1 \mathrm{~K}$ & $1.75 \$-1 \mathrm{~K}$ \\
\hline Sleep & $1 \mathrm{uA}$ & $1 \mathrm{uA}$ \\
\hline
\end{tabular}

\subsection{Device Operation}

Proposal processing method is that individual controls were performed with on/off about $\mathrm{ADC}$, SPI and I2C, if necessary. It is possible to achieve efficient energy utilization efficiency as shown in Figure 5.
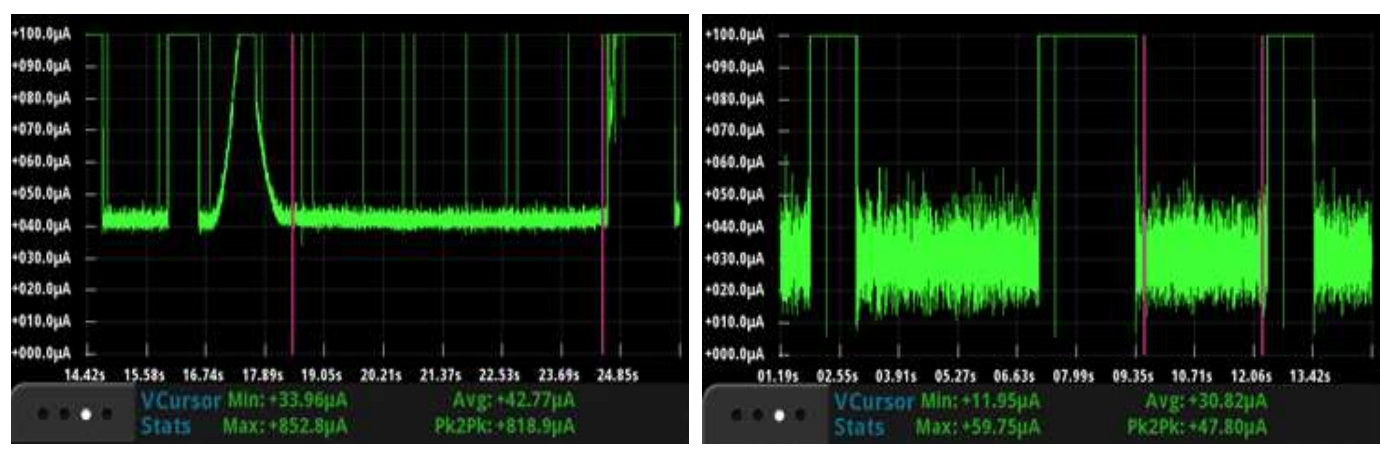

Figure 5. Experiment Results about Event-driven Approach: (Left) General Approach, (Right) Proposed Approach

\subsection{Sensor Operation}

The developed device has a multi-sensor applied to temperature, acceleration, magnetic field, UV, and US. The general IoT devices that do not use this proposed method process them sequentially. When the number of sensors was increased, the processing time and the energy consumption were also increased. On the other hand, the operation time in the proposed method was shortened. As a result, the power consumption can be reduced by 4 times as shown in Figure 6.
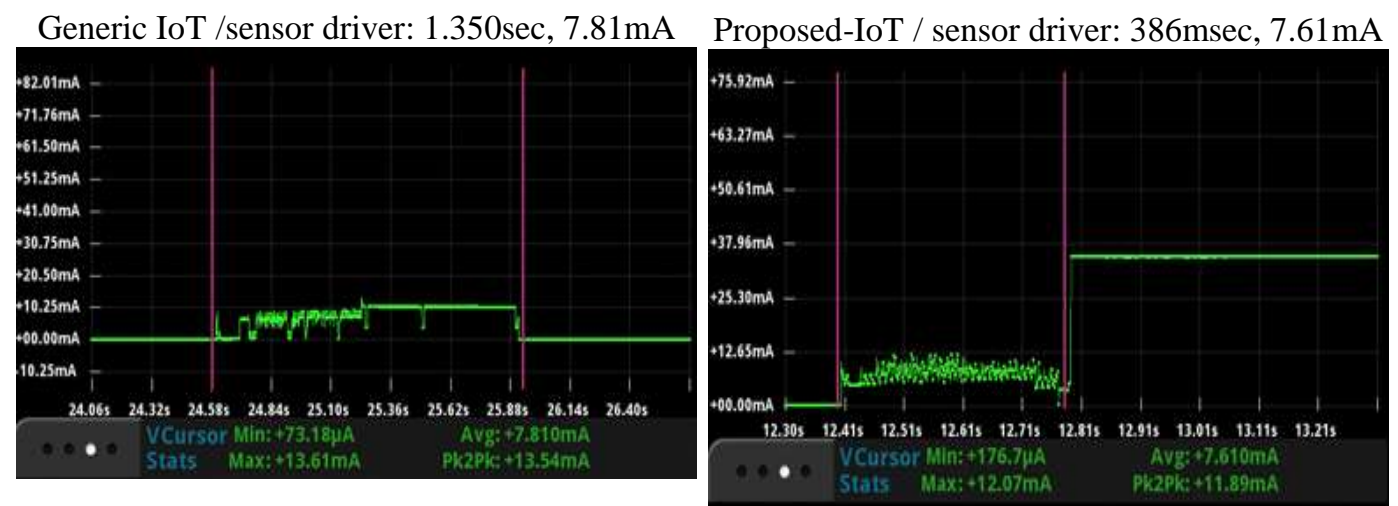

Figure 6. Experiment Results about Composite Sensor:

(Left) General Approach, (Right) Proposed Approach 


\subsection{Data Transmission}

Assuming that LoRa technology with SF9, Power 14, and PA_BOOST on is applied, the general IoT devices are CPU On by Polling method at RF TX. On the other hand, the proposed device is CPU Off by RF Interrupt method. As a result, the transmission packet is long but the TX power is low in Figure 7.

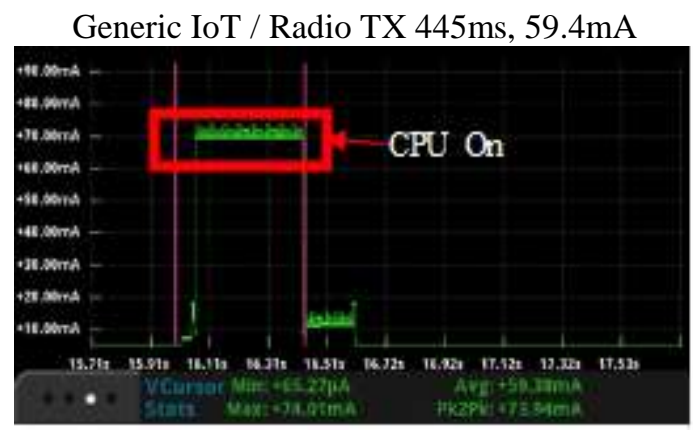

Proposed-IoT / Radio TX: 571ms, 34.24mA

\section{Figure 7. Experiment Results about Data Transmission:} (Left) General Approach, (Right) Proposed Approach

\subsection{Operation Results}

Through the experimental results, the proposed scheme was $2.0146 \mathrm{mAh} / 1$ day and the general IoT device was $2.3747 \mathrm{mAh} / 1$ day. It has a relatively low consumption of $85 \%$. As a result, it can be operated for about 41 months and 35 months, respectively. So the battery operation period will relatively longer due to the improvement of power consumption using the proposed technology.

\section{Table 3. Comparison of Consumption Energy}

\begin{tabular}{|c|c|c|c|c|c|c|}
\hline & \multicolumn{3}{|c|}{ Generic IoT } & \multicolumn{3}{c|}{ Proposed IoT } \\
\hline & 1 Day(mAh) & Year(mAh) & Ratio(\%) & 1 Day(mAh) & 1 Year(mAh) & Ratio(\%) \\
\hline LoRa Tx & 0.3524 & 128.64 & 14.8 & 0.4522 & 165.06 & 22.4 \\
\hline LoRa Rx & 0.0293 & 10.69 & 1.2 & 0.0161 & 5.89 & 0.8 \\
\hline LoRa Retry & 0.6506 & 237.46 & 27.4 & 0.7356 & 268.49 & 36.5 \\
\hline Sensor Driver & 0.1406 & 51.31 & 5.9 & 0.0392 & 14.30 & 1.9 \\
\hline CMD Periodic & 0.1716 & 62.63 & 7.2 & 0.0281 & 10.25 & 1.4 \\
\hline Sleep & 1.0302 & 376.04 & 43.4 & 0.7434 & 271.34 & 36.9 \\
\hline Total & 2.3747 & 866.76 & 100.0 & 2.0146 & 735.32 & 100.0 \\
\hline Life & & 2.95 (Year) & & & 3.47 (Year) \\
\hline
\end{tabular}

\section{Conclusion}

This paper designed an operation algorithm for lightweight devices powered by small battery and working in resource-constrained environments. Designed technologies include ultra-low-power embedded OS design, minimization of processing time through parallel processing, event-driven operation algorithm through sleep and interrupt method, simple registration to complete registration with one packet, and a hybrid sensor technique. The proposed algorithm was implemented based on the M0 CPU, and it can be confirmed that the power consumption was improved by $10 \sim 30 \%$ compared with the general IoT device. 


\section{Acknowledgments}

This paper is a revised and expanded version of a paper entitled "Implementation of Lowe Power System in IoT Service Environment", submitted to HSST April 2018 Workshop.

\section{References}

[1] oneM2M TS-0001-V1.10.0: "Functional Architecture".

[2] IETF RFC 7252: "The Constrained Application Protocol (CoAP)".

[3] Lightweight Machine to Machine Technical Specification Draft version 1.0 -10 Sep, (2015).

[4] M. Hye Park, Y.-H. Kim and Seung-Bae Lee, "Internet-based Power Information Network Design Study", Korean Institute of Communications and Information Sciences, (2015) November, pp. 228-230.

[5] M. Hye Park, Y.-H. Kim, S.-B. Lee, B.-s. Lee and M.-H. Kim, "Apparatus and Method for Generating Address of System of Internet of Thing”, Korea. Patent 1020160133731, (2016) October 14.

[6] Telecommunications Technology Association Group Standard TTAK.KO-10.1034, Base Specifications for IoT (Internet of Things) Services in Electricity and Energy Domain, (2017) December.

[7] R. Es-sadaoui, L. Azergui, Y. Ghanam and J. Khallaayoune, "Design and experimentation of a low-power IoT embedded system for wireless underwater sensing", Institute of Electrical and Electronics Engineers, (2017).

[8] LoRaWAN Regional Parameters, LoRa Alliance Inc., San Ramon, CA, Ver.1.0, (2016) July.

\section{Authors}

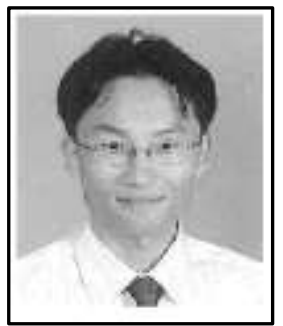

Younghyun Kim, he received B.Eng. degree in information and telecommunication engineering from Korea Aerospace University (KAU), Korea, in 2002, and M.S. degree in information and communications from Gwangju Institute of Science and Technology (GIST), Korea, in 2004. Since 2004, he has been a researcher of the Korea Electric Power Corporation. His current interests are wired/wireless communication system design, analysis, and implementation in IoT including utility automation.

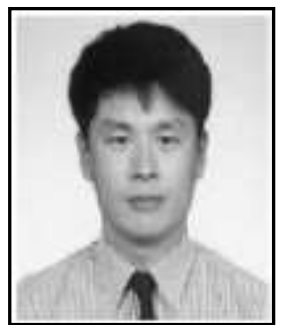

Myong-Soo Kim, he received M.S. degree in Electric Engineering and Ph.D. degree in Computer Science and Engineering at Pennsylvania State University, University Park, USA, in 2008 and 2011, respectively. Since 1996, he has been a researcher of the Korea Electric Power Corporation. His special fields of interest are utility automation and communication systems in Smart Grid including utility protocol and security.

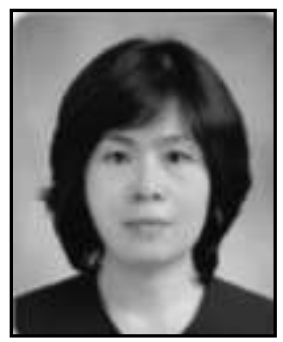

Myong-Hye Park, she received B.S degree and M.S. degree in Electroic Engineering at Kyungpook National University in 1993 and 1995, respectively. Since 1995, she has been a researcher of the Korea Electric Power Corporation. Her special fields of interest are communication network technologies and Internet of Things. 


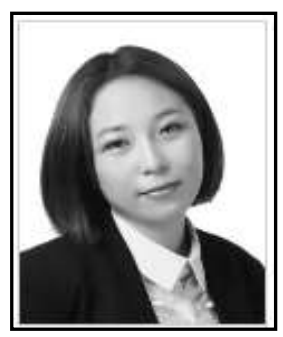

Soo-Kyong Kang, received the B.Eng. degree in information communication technology from Hanyang University in 2008. She is a researcher at the Research Institute of Korea Electric Power Corporation. Her research interests include communication system and IoT in Energy Environment.

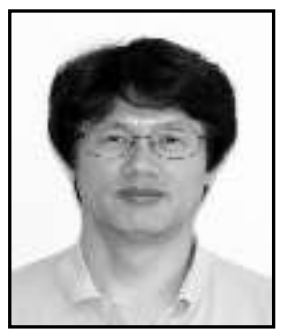

Chang-Soo Eun, he received M.S. degree in Electric Engineering at Seoul National University in 1987 and Ph.D. degree in Electric Engineering and Computer Science at Texas Austin State University, USA, in 1995. Since 1997, he has been a professor of Chungnam National University. His special fields of interest are Communication System and Signal Processing in Smart Grid including utility protocol and security. 